\title{
Mapping Attention Across Multiple Media Tasks
}

\author{
Jacob T. Fisher ${ }^{1}$, Frederic R. Hopp², \& René Weber ${ }^{3^{*}}$ \\ [1] Institute of Communications Research, Charles H. Sandage Department of Advertising College of Media, University of Illinois Urbana- \\ Champaign [2] Amsterdam School of Communication Research [3] Media Neuroscience Lab, Department of Communication, University of \\ California Santa Barbara
}

\begin{abstract}
Successful media processing requires that an individual attend to relevant information embedded among numerous competing options. Although the factors that drive individuals' attention toward or away from a single media processing task are relatively well characterized, there is a lack of understanding regarding how the allocation of attention proceeds in the presence of multiple, concurrent tasks. In four experiments, we show that attention during media processing is contingent on features of the "primary" task, but also on features of other available tasks. Specifically, we find evidence suggesting that "secondary" tasks during media processing elicit more attention when they are more rewarding and that the attention-capturing influence of these tasks is magnified when the "primary" task is more cognitively effortful. These results provide support for recent theoretical advancements in media psychology research and point to promising future directions using models of motivated attention to predict the allocation of attentional resources across multiple concurrent media tasks.
\end{abstract}

Keywords: attention, motivation, reward, media multitasking, message processing

Communication is dynamic, information-rich, and often occurs across multiple modalities and channels simultaneously (Lang, 2000; Lang \& Ewoldsen, 2009). Because human beings can only process a finite amount of information at any given time (e.g., Hasher \& Zacks, 1979; Shiffrin \& Schneider, 1977), an individual must decide (either consciously or unconsciously) what information should be selected for processing and what should be filtered out. This selection process is often referred to as attention (Broadbent, 1958). Attention has been called the sine qua non of message reception (Cartier \& Harwood, 1958), and is a precursor, moderator, or outcome of interest in a myriad of communication theories. As such, understanding how, when, and why humans direct their attention to communication and media tasks has been a core question for communication researchers for decades (Chaffee \& Berger, 1987).

In this time, communication scientists have made much progress toward understanding how certain features of tasks and messages influence attention, as well as how attention influences outcomes of interest like memory, enjoyment, or persuasion (for a recent review, see Fisher et al., 2018a). At present, though, it is unclear to what extent models describing how people pay attention to a single, isolated media task can be used to understand how people allocate attentional resources across multiple, concurrent tasks. This gap in current knowledge is especially salient given the recent rise of media multitasking (Segijn et al., 2018; Wang et al., 2015), now the de facto mode of media consumption in the industrialized world (Deloitte, 2017).

To address this gap, we begin with a review of recent advancements in attention research that conceptualize attention in terms of a "priority map" that integrates top-down, bottom-up, and motivational signals to guide information processing (Failing \& Theeuwes, 2018; Zelinsky \& Bisley, 2015). We contextualize this literature in view of recent updates to the limited capacity model of motivated mediated message processing (LC4MP; Fisher et al., 2018a, 2018b; Lang, 2000, 2009), to generate predictions regarding how individuals will direct attention within and between concurrent media tasks. We focus specifically on the roles of reward and cognitive load, hypothesizing that more rewarding media tasks will elicit more attention and that increasing cognitive load in a "primary task" will both: a) reduce attentional performance across all tasks, and b) will magnify the attention-capturing of rewarding alternative tasks. We test these hypotheses across four experiments using a novel experimental paradigm that directly manipulates reward and cognitive load across multiple concurrent media tasks while controlling for potential confounds. ${ }^{1}$

1. All code and data from this experiment are available on OSF https://osf.io/49673/?view_only=a3b043c03f61497e81ea5fe7b032305d. 


\section{From a Spotlight to a Map}

Individuals direct their attention using a suite of neural and behavioral processes developed over evolutionary time to enable efficient acquisition of relevant information from the environment (Lang, 2000, 2009; Weber et al., 2009). Early models described attention as a "spotlight" or a "zoom lens" (Eriksen \& St. James, 1986; Posner et al., 1980) with a single, indivisible focal point and a central capacity limitationusually related to working memory (Baddeley \& Hitch, 1974). Attention was described as either "top-down" (deliberate, based on current goals) or "bottom-up" (pre-conscious, driven by stimuli in the environment; Corbetta \& Shulman, 2002; Desimone \& Duncan, 1995). However, mounting evidence over the last two decades indicates that this "spotlight" model of attention falls short in a number of ways. Most notably, it has become clear that attention can be distributed across multiple items at the same time (Awh \& Pashler, 2000; McMains \& Somers, 2004), and seems to be strongly influenced by associative learning processes that blur the boundaries between what is "top-down" and what is "bottom-up" (Awh et al., 2012).

These findings have catalyzed the adoption of a new framework in which attention is described in terms of a "priority map" that integrates top-down, bottom-up, and (learned) motivational signals into a common representational space (Bisley \& Mirpour, 2019; Fecteau \& Munoz, 2006; Zelinsky \& Bisley, 2015). The topography of this map serves to guide attention among potential targets to resolve conflict and direct information processing (Failing \& Theeuwes, 2018). Peaks of neural activation on the priority map correspond to spatial locations (or features) that are more likely to be attended, predicting orienting responses, visual saccades, and other forms of attentional resource allocation (B. A. Anderson et al., 2011; Chelazzi et al., 2014; Ptak, 2012), as well as memory for certain stimuli over other ones (Fine \& Minnery, 2009; Zelinsky \& Bisley, 2015).

Evidence indicates that the attentional priority of a given stimulus is a product of three interacting factors. The first of these is salience, or the extent to which the stimulus is brighter, louder, or otherwise distinct from surrounding stimuli (Itti \& Koch, 2001). The second is the goal-relevance of stimulus features (Corbetta \& Shulman, 2002; Theeuwes, 2010, 2019). This is most clearly demonstrated in the phenomenon of "attention blindness," in which stimuli that would normally be very noticeable go almost completely unattended in the presence of a salient goal (e.g., Simons \& Chabris, 1999). Finally, attention to a stimulus is influenced by its (learned) associations with previous rewarding or punishing outcomes, in a way that is largely independent of currently held goals (Chelazzi \& Santandrea, 2018). Reward- or punishmentassociated stimuli robustly and persistently "capture" attention even when they are not salient or relevant to current goals (B. A. Anderson, 2016; Failing \& Theeuwes, 2018).

This value-driven modulation of attention is undergirded by neural signals that encode the expected reward that will be attained by selecting a given behavior in view of a particular goal (Floresco et al., 2008; Rangel \& Hare, 2010). These neural signals are largely analogous to one another across a wide range of value-related stimuli, including love, money, information, and material goods (Gu et al., 2019; Kobayashi \& Hsu, 2019). Additional work has shown that there are also neural signals that seem to serve to "discount" the reward value of a given option as a function of the costs (e.g. opportunity cost, effort, or threat; Shenhav et al., 2013; Westbrook \& Braver, 2015) that may be incurred if it is selected. As the cost of a given option increases relative to its reward value, other options with a more satisfactory balance of reward and cost become more likely to be chosen. It is theorized that this "common currency" (Levy \& Glimcher, 2012) of reward and cost enables the brain to compute the marginal value of a diverse array of stimuli and to redraw the priority map in favor of relatively more valuable options (Grabenhorst \& Rolls, 2011).

Critically, these "priority maps" are not unique to early stages of attentional processing. In fact, they have been observed throughout the cortical hierarchy (Behrens et al., 2018; Wilson et al., 2014) and seem to be instrumental in guiding even very complex selection processes (Chelazzi \& Santandrea, 2018; Zelinsky \& Bisley, 2015). These findings have begun to blur the lines between the (historically largely separate) fields of attention, decision making, and cognitive control research, and have produced growing consensus that attention may be merely an example of a domain-general neural process through which organisms select certain behaviors over other ones in order to reach their goals (B. Anderson, 2011; Gottlieb \& Balan, 2010; Krauzlis et al., 2014). 


\section{Maps and concurrent media processing tasks}

These findings have begun to be contextualized within the communication and media literature in a recent update to the Limited Capacity Model of Motivated Mediated Message Processing (LC4MP; Fisher et al., 2018b). In the LC4MP, it is proposed that attentional performance in a secondary task (usually operationalized as reaction time to an auditory and/or visual probe) during media processing is an index of resources available-cognitive resources that were allocated to the primary task that were not required to successfully process it (Fox et al., 2007; Lang, 2009; Lang et al., 2006). This conceptualization has served to greatly increase our understanding of how people pay attention to single messages but also limits the ability of the model to generate predictions in media multitasking situations, in which "secondary" task performance may depend on characteristics of the secondary task itself (e.g. its salience, goal-relevance, or reward), rather than just on characteristics of the primary task.

Incorporating the updated conceptualization of attention outlined above into the LC4MP provides the theoretical scaffolding necessary to update the model so that it can better account for attention during multiple concurrent media tasks. Recall that rather than assuming that attention is a "spotlight" with a single, central focus, this framework assumes that attention can be proportionally allocated to multiple items in parallel and that this allocation process is guided by neural signals encoding the (relative) attentional priority of each item. It follows, then, that attentional resources need not "pass through" the primary task to the secondary task, as currently described in the LC4MP. Instead, it can be proposed that: resources are concurrently distributed across all available tasks as a function of their relative priority, and that attentional performance in any given task is an indicator of the resources allocated to the task minus the resources required to successfully complete it.

These theoretical propositions generate a few central predictions: First (H1), more rewarding media tasks should elicit more attention. Second (H2), as cognitive load (i.e. the effort or costs required to complete a task) increases, overall attentional performance should diminish, reflective of a reduction in available cognitive resources. Third (H3), as a given task increases in cognitive load, the attention-capturing influence of rewarding alternative tasks should be magnified, reflective of a change in the relative reward value of the "primary" task as compared to the rewarding alternative. Finally (H4), Once a critical threshold is reached, resources should be allocated away from the primary task and toward the secondary task. Herein, we test these predictions (and rule out potential confounds) across four experiments designed to investigate how reward and cognitive load in concurrent media processing tasks act and interact to influence the allocation of attention within and across each task.

\section{Methods}

\section{General overview}

Four behavioral experiments were conducted to investigate the influence of reward and cognitive load on the allocation of attention across multiple concurrent media processing tasks. In each experiment, participants played Asteroid Impact, ${ }^{2}$ an interactive, open-source computer game programmed in Python. Playing Asteroid Impact involves using the mouse to move a spaceship around the screen to collect crystals and avoid crashing into quickly moving asteroids. Participants also were asked to complete a concurrent task, in which they pressed a button on a computer keyboard when they saw a particular shape appear on the screen. These reaction time (RT) probes appeared in a randomized position on the screen each time they were generated and were accompanied by an auditory tone. ${ }^{3}$ In order to encourage meaningful participation, participants were told that the top three scorers in the game overall would receive a $\$ 50$ gift card. The game was presented in a lab setting on Dell 1600 x 900 monitors with a $60 \mathrm{~Hz}$ refresh rate. Audio was presented through Bose QuietComfort 15 headphones with computer volume set to 15/50. All stimuli and procedures were approved by the human subjects committee of the university.

\footnotetext{
2. https://github.com/asteroidimpact/asteroid_impact_py3

3. The frequency, duration, and required keypress for the secondary task varied slightly from experiment to experiment. These differences are outlined within the sections devoted to each experiment.
} 


\section{Participants}

Participants in all four experiments were drawn from the human subjects pool at a large university in the western United States (for final n's and relevant demographic information for each experiment, see Table 1). Recent work has revealed considerable variability in effect sizes across studies in communication research (Rains et al., 2018). As such, a small effect size was assumed (Cohen's $d=.2$ ). A power analysis was conducted using the simr package in $\mathrm{R}, 4$ revealing that a sample of 60 participants would be sufficient for $80 \%$ power to detect such an effect (Green \& MacLeod, 2016). As such, we were sure to recruit at least 60 participants for each study.

\begin{tabular}{ccccc}
\hline & $\mathrm{n}$ & Mean Age (SD) & \% Female & Mean Video Game Skill (SD) \\
\hline Experiment 1 & 123 & $19.79(1.38)$ & 62.60 & $3.64(1.67)$ \\
Experiment 2 & 238 & $19.59(1.40)$ & 70.60 & $3.61(1.56)$ \\
Experiment 3 & 89 & $19.98(2.04)$ & 71.91 & $3.94(1.54)$ \\
Experiment 4 & 65 & $19.59(1.42)$ & 61.54 & $3.93(1.55)$ \\
\hline
\end{tabular}

Table 1: Summary statistics describing final subject pools in each of the four experiments.

\section{Variables}

Manipulating cognitive load. Cognitive load was manipulated in all experiments by including a 1-back working memory maintenance task into the crystal collection portion of the video game. These tasks have been widely used and validated within the cognitive effort literature (see e.g., Veltman et al., 2003). Participants were told that collecting two of the same colored crystal in a row (e.g. collecting a blue crystal and then immediately collecting another blue crystal) would result in a loss of 1000 points. This required the participants to maintain the identity of the crystal they had just collected in working memory while pursuing other crystals, and to constantly update the identity of the last crystal collected, thus loading working memory and increasing the complexity of the primary task.

Manipulating reward. In all experiments, reward was manipulated by assigning varying point values to the probes that were presented in the RT task. In experiments 1 and 2, the rewardingness of the RT task was manipulated on a trial-by-trial basis within each round, with high and low reward probes presented at random within the same round. This allowed us to test the influence of reward on attentional resource allocation within the secondary task. In experiments 3 and 4, the rewardingness of the RT task was manipulated between rounds, such that every probe presented in a given round was either high or low reward. Changing the overall rewardingness of the secondary task at this longer timescale allowed us to test the influence of reward on attentional resource allocation across the two tasks. In all experiments, the high reward probe was worth 1000 points and the low-reward probe was worth 10 points (for a visual depiction of reward conditions, see Figure 1).

Measuring attention and task performance. Recall that participants were asked to complete two concurrent tasks: a crystal collection task and a reaction time task. In each experiment, task performance within the crystal collection task was calculated by summing the number of crystals that a participant collected within each thirty-second window of gameplay and then dividing that number by the number of times that the subject crashed into an asteroid during that window. Performance was calculated for each participant and each 30-second window in each round and each of these observations was treated as one data point within the linear mixed-effects model described below. This measure was approximately equally distributed across all four experiments. Performance in the RT task was recorded as the interval (in milliseconds) between when the probe first appeared on screen and when the appropriate button

4. https://cran.r-project.org/web/packages/simr 
was pressed. Each probe remained on screen for a maximum of ten seconds. If the incorrect button was pressed or the probe remained on the screen for more than ten seconds, the trial was recorded as a miss.

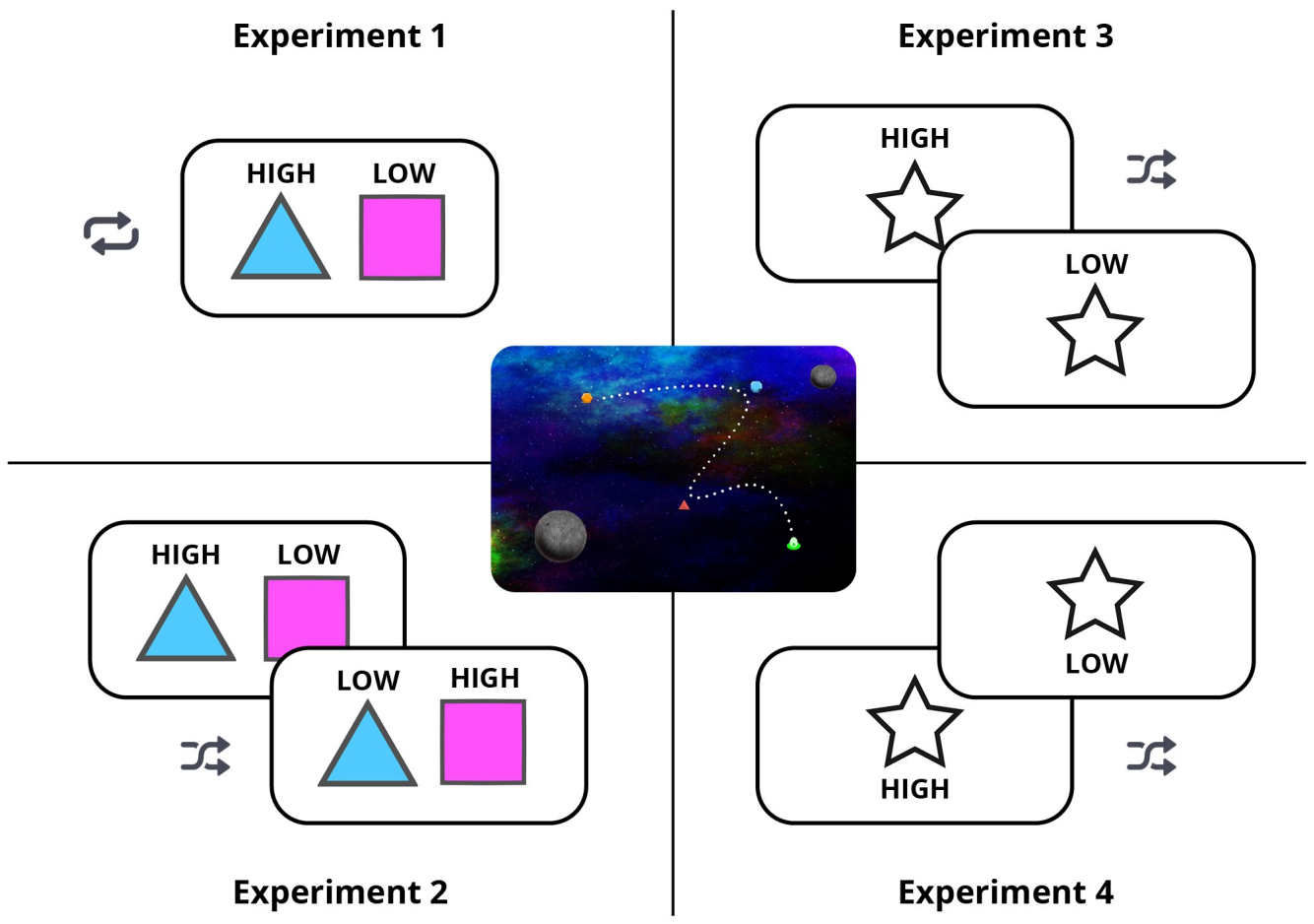

Figure 1: Schematic of reward conditions across all four experiments. In all conditions, the primary task was to navigate a space ship around the screen and collect crystals while avoiding asteroids. In all experiments, there was also a secondary task in which participants were asked to press a key when they see a particular shape (a reaction time probe) appear on screen. In Experiment 1, high and low reward RT probes were present within the same condition, and were consistent across the entire game play period. In Experiment 2, high and low reward RT probes were present within the same condition, but the value of each of the two probes was switched in between levels such that each probe was sometimes high reward and sometimes low reward. In experiments 3 and 4, there was only one type of RT probe within each condition (a white star), and the probe was either high reward or low reward. In experiment 4, RT probes were much more frequent, occurring once every $\sim 2$ seconds as opposed to once every $\sim 10$ seconds.

\section{Analysis procedures}

All frequentist analyses were conducted using linear mixed-effects modeling. Models were created using the Ime4 package (Bates et al., 2015) in the R statistical computing language (R Core Team, 2013). ${ }^{5}$ Cognitive load, reward, and the interaction between load and reward were treated as fixed effects in each model. Random intercepts and slopes were included for each participant and for each experimental condition nested within each participant.

As an additional test of the hypotheses put forward in this study, Bayesian analyses were also conducted (Morey et al., 2016). ${ }^{6}$ The primary coefficient of interest in a Bayes factor analysis is the Bayes factor $\left(\mathrm{BF}_{10}\right)$, which gives an indication of the likelihood that a model $\left(\mathrm{M}_{\mathrm{A}}\right)$ is true (compared to a particular null model, $M_{0}$ ) given the evidence provided by the data. For example, if $M_{A}$ has a $B F_{10}$ of 8 , this means that the data suggest $\mathrm{M}_{\mathrm{A}}$ is about 8 times more likely to be correct than $\mathrm{M}_{0}$ given the data observed in the study. For each

5. A more precise list of the computational environment used for these analyses is available on the OSF repository for this project https://osf. io/49673/?view_only=a3b043c03f61497e81ea5fe7b032305d.

6. All Bayesian analyses were conducted using the $\operatorname{lmBF}$ ( ) function in the BayesFactor package in R (Morey et al., 2018). Note that Bayes factors in factorial designs must be numerically estimated via monte-carlo simulations (Rouder et al., 2017). As such, a replication analysis with the provided data will lead to Bayes factors that vary slightly within the reported range. 
of the main effects of interest, the null model consisted of only random effects. For the interaction effects, the null model consisted of the random effects and the main effects of each of the variables of interest. All Bayesian analyses are reported in Tables 2 and 3.

\section{Experiment 1}

\section{Methods}

A total of 123 participants participated in Experiment 1. In this experiment, participants played 19 minutes of Asteroid Impact. There was a 1-minute practice round followed by one six-minute round of gameplay in each of three conditions (baseline, perceptual load,7and cognitive load). All rounds except the practice round were presented in randomized order. In this experiment, one RT probe (a blue triangle) was associated with low reward and the other (a purple square) was associated with high reward.

\section{Results}

Reward. We predicted that high reward would increase the amount of attentional resources allocated to a task, reflected in reduced RTs relative to low-reward stimuli. As high and low reward RT probes were intermingled in this experiment, we did not expect any influence of reward on the crystal collection (primary) task performance. These predictions were supported (See Figure 2.1c, Table 2). Participants responded faster to the high reward probes $(\mathrm{M}=923.16, \mathrm{SD}=163.90)$ compared to the low reward probes $(\mathrm{M}=1092.94, \mathrm{SD}=215.14)$.

Cognitive load. It was predicted that increased cognitive load would result in decreased availability of attentional resources, leading to longer RTs and reduced task performance. These predictions were supported (See Figure 2.1a, b, Tables 2, 3). In the baseline condition participants' RTs were faster (M $=932.59, \mathrm{SD}=186.30)$ than in the cognitive load condition $(\mathrm{M}=1157.84, \mathrm{SD}=240.41)$. Participants also performed better in the crystal collection task in the baseline condition $(\mathrm{M}=33.93, \mathrm{SD}=3.64)$ than in the high load condition $(\mathrm{M}=24.51, \mathrm{SD}=4.55)$.

Interaction between reward and load. We also predicted an interaction between reward and cognitive load such that the gap between high and low reward RT probes should be larger when cognitive load was higher, reflecting prioritization of high-reward stimuli as the "cost" of attending to the task increased. This prediction was supported (see Figure 2.1d, Table 2). The distance between high and low reward RT probes was larger in the high load condition $(\Delta \mathrm{M}=397.85)$ than in the baseline condition $(\Delta \mathrm{M}=139.06)$.

\section{Discussion}

In this experiment, we showed that participants responded faster to high-reward RT probes compared to low-reward RT probes, supporting H1. We also showed that increased cognitive load led to decreased performance both in the crystal collection task and in the reaction time task, suggesting an overall decrease in the availability of attentional resources as the cognitive effort required to complete the crystal (primary) task increased, supporting H2. Finally, we showed that the gap between high- and low-reward RTs is magnified whenever cognitive load is high, supporting H3.

\section{Experiment 2}

\section{Methods}

Experiment 2 was designed to replicate findings from Experiment 1 in a larger sample and to test the prediction that observed responses to differently rewarding probes are the result of learning and updating value representations rather than on other stimulus-specific features known to drive attention (e.g. differences in sensory salience between the probes). In total, 238 participants completed Experiment 2. This experiment was identical to Experiment 1, with two exceptions. First, rather than one of the RT probes

7. Perceptual load was manipulated in each experiment in view of other research questions, but as perceptual load is not relevant to the hypotheses presented herein, these data are not further reported. 
being high reward and the other being low reward throughout the experiment, the value of each of the probes switched in between each round of gameplay. This means that a different probe (either the square or triangle) was of high value in each round. Second, to accommodate the extra rounds needed to switch probe values between each round, participants played two five-minute rounds in each load condition rather than one six-minute round.

\section{Results}

Reward. Also replicating Experiment 1, RTs were faster for the high reward probe than for the low reward probe (See Figure 2.2c, Table 2). Despite the fact that the identity of the high-value probe was switched each round, the high reward probes elicited faster $\mathrm{RTs}(\mathrm{M}=839.47, \mathrm{SD}=163.89)$ than did the low reward probes $(M=1030.14, S D=269.48)$.

Cognitive load. Replicating Experiment 1, increased cognitive load was shown to reduce overall attentional resource availability, leading to reduced task performance and lengthened RTs (See Figure 2.2a, b, Table $2,3)$. RTs were slower in the high load condition $(M=1030.14, S D=269.48)$ compared to the baseline condition $(\mathrm{M}=839.48, \mathrm{SD}=163.89)$. Participants also performed worse in the crystal collection (primary) task in the high load condition $(\mathrm{M}=22.26, \mathrm{SD}=6.17)$ than they did in the baseline condition $(\mathrm{M}=30.83, \mathrm{SD}$ $=5.80)$.

Interaction between load and reward. Even though the identity of the high-reward probe was switched between rounds, an interaction between load and reward was still observed (See Figure 2.2d, Table 2). In the baseline condition, the RT gap between high and low reward probes was $73.75 \mathrm{~ms}$, but this gap expanded to $253.59 \mathrm{~ms}$ in the high load condition.

\section{Discussion}

In this experiment we replicated the findings from Experiment 1, supporting H1-3 in a larger sample and providing evidence that the effects we observed in Experiment 1 were not confounded by differences in sensory features of the stimuli. These results provide clear evidence for the priority-driven nature of attentional resource allocation across stimuli within a particular task-between high- and low-reward RT probes. It remains unclear, however, whether this priority map approach can also account for situations in which resources are allocated away from a given media task in favor of another, concurrent task, as is commonly observed in communication and media research. As such, experiments 3 and 4 were designed to manipulate the overall rewardingness of the RT task relative to the crystal collection task.

\section{Experiment 3}

\section{Methods}

A total of 92 participants were included in experiment 3. Participants completed a 1-minute practice round followed by two six-minute rounds of gameplay in each of three conditions (baseline, perceptual load8, cognitive load). All rounds except the practice round were presented in random order. In the RT task, participants were asked to press the spacebar when they see a probe appear on the screen (a white star). One RT probe was presented at random within each 10-second block of gameplay. Probes remained on screen for a maximum of 10 seconds before disappearing. As mentioned above, in this experiment, the rewardingness of the RT probes was manipulated between rounds, allowing us to investigate how performance in the crystal collection task is influenced relative to the overall rewardingess of the RT task. In one of the rounds within each load condition, responding to the RT probe was worth 1000 points (high reward), and in the other round, it was worth 10 points (low reward).

8. Again, perceptual load was manipulated in each experiment in view of other research questions, but as perceptual load is not relevant to the hypotheses presented herein, these data are not further reported. 


\section{Results}

Reward. The main effect of reward on RT observed in Experiments 1 and 2 was replicated in Experiment 3 (See Figure 1.3e, Table 2). Participants responded faster to high reward probes $(\mathrm{M}=701.95, \mathrm{SD}=147.38$ ) compared to low reward probes $(\mathrm{M}=1022.03, \mathrm{SD}=871.99)$. Analyses also revealed a significant (although very small) effect of reward on primary task performance such that participants performed worse in the crystal collection task when the RT task was high reward (See Figure 2.3b, Table 3).

Cognitive load. The main effect of cognitive load on both RT and performance was replicated (See Figure 2.3a, d, Table 2, 3). Participants responded more quickly to RT probes in the baseline condition $(\mathrm{M}=666.82$, $\mathrm{SD}=133.34)$ than in the high load condition $(\mathrm{M}=951.85, \mathrm{SD}=395.21)$. Participants also performed worse in the crystal collection task in the high load condition $(\mathrm{M}=22.55, \mathrm{SD}=4.49)$ than they did in the baseline condition $(\mathrm{M}=30.58, \mathrm{SD}=2.99)$.

Interactions between load and reward. Replicating Experiments 1 and 2, the gap in response time between high and low reward RT probes was wider when cognitive load was high $(\Delta \mathrm{M}=524.47)$ than when load was low ( $\Delta \mathrm{M}=147.62$; See Figure 2.3f, Table 2). It was also predicted that load and reward would interact to influence primary task performance such that the presence of high reward RT probes should reduce primary task performance to a greater extent when cognitive load was high. This prediction was also supported (See Figure 2.3c, Table 3).

\section{Discussion}

In Experiment 3, we replicated the findings of Experiments 1 and 2 regarding the main effects of reward and load (H1-2), and also the interaction between load and reward on attentional priority (H3). Results from Experiment 3 also extend the findings of Experiments 1 and 2 in showing that when the overall rewardingness of the RT task increased, performance in the crystal collection task decreased and that this effect is magnified when the crystal collection task was more cognitively effortful. This supports H4 and suggests that decreased RTs observed for high-reward probes is due to attentional resources being reallocated from the crystal collection task in order to respond more quickly to the rewarding RT task.

\section{Experiment 4}

\section{Methods}

A total of 65 participants completed Experiment 4. This experiment was designed to replicate the findings of Experiments 1, 2 and 3 and to investigate whether increasing the frequency with which participants had to respond to the RT task (thus further increasing the attentional resource requirements of the task) would magnify the effects that were observed in Experiment 3, further supporting H3 and H4. This experiment was identical in design to Experiment 3, with the exception that the RT probes appeared once per 3-second window of gameplay rather than once per ten-second window. Probes remained on screen for a maximum of 2 seconds before disappearing.

\section{Results}

Reward. All main effects of reward were replicated in this experiment (See Figure 2.4e, Table 2). Participants responded faster to high reward $\mathrm{RT}$ probes $(\mathrm{M}=642.84, \mathrm{SD}=158.41)$ than to low reward RT probes $(\mathrm{M}=$ 1041.27, SD = 297.66). Participants also performed worse in the crystal collection task whenever RT probes were more rewarding $(\mathrm{M}=28.98, \mathrm{SD}=5.28)$ compared to when they were less rewarding $(\mathrm{M}=31.32, \mathrm{SD}=$ 5.03; See Figure 2.4b, Table 3).

Cognitive load. All main effects of load were replicated (See Figure 2.4a, d, Table 2, 3). Participants responded slower to the RT probes when cognitive load was high $(M=866.05, S D=209.04)$ than they did in the baseline condition $(\mathrm{M}=680.50, \mathrm{SD}=163.95)$. Participants also performed worse in the crystal collection when load was high $(\mathrm{M}=23.51, \mathrm{SD}=5.89)$ compared to the baseline condition $(\mathrm{M}=36.24, \mathrm{SD}=4.38)$.

Interactions between load and reward. Replicating Experiment 3, we predicted an interaction between cognitive load and reward on both performance and RTs. This prediction was supported (See Figure 2.4c, $\mathrm{f}$, 
Table 3). In the baseline condition, participants performed similarly in the crystal collection task whether the RT task was high or low in reward $(\Delta \mathrm{M}=1.47)$, but in the high load condition, participants' performance in the crystal collection task dropped when the RT task was high reward $(\Delta \mathrm{M}=8.72)$. An interaction between cognitive load and reward on RTs was not observed in this experiment, a result which we discuss below.

\section{Discussion}

In this experiment, we replicated the main effects (H1-2) observed in Experiments 1-3. We also extended the findings of Experiment 3 by showing that increasing the resource requirements of a rewarding alternative task can drive individuals to allocate attentional resources away from a "primary" task, and toward the alternative task especially when the primary task requires a great deal of cognitive effort (supporting H4). The lack of an observed interaction between cognitive load and reward on RTs, coupled with the large decrease in crystal collection performance when load and reward are both high suggests that some participants may have entirely abandoned the crystal collection task in favor of the RT task in this condition, allowing them to respond rapidly to probes regardless of the load requirements of the primary task. Indeed, we see intimations that this may be the case (see Figure 2d). A clear subgroup of participants shows quite low performance in the crystal collection task when load and reward are both high. If these participants simply reallocated (or offloaded) attentional resources from the crystal collection task to the RT task in this condition, RT patterns would be expected to resemble those observed here. These results indicate that task priority is contingent upon the balance of reward and effort across concurrently available media tasks and that when this balance is interrupted (i.e. when load is high in one task and reward is high in another), participants will reallocate attentional resources away from the cognitively demanding task and toward the rewarding one.

\begin{tabular}{|c|c|c|c|c|c|}
\hline Manipulation & Experiment & $(\mathrm{DF}), \beta$ & $95 \%$ CI & Cohen's $d$ & $\mathrm{BF}_{10}$ \\
\hline \multirow[t]{4}{*}{ Cognitive Load } & 1 & $(1,122), .222^{\star \star \star}$ & {$[.185, .260]$} & 0.452 & $2.29 \times 10^{5}( \pm 2.43 \%)$ \\
\hline & 2 & $(1,237), .157^{\star \star *}$ & {$[.132, .182]$} & 0.328 & $1.79 \times 10^{4}( \pm 1.44 \%)$ \\
\hline & 3 & $(1,88), .314^{\star \star *}$ & {$[.265, .363]$} & 0.665 & $1.54 \times 10^{8}( \pm 1.09 \%)$ \\
\hline & 4 & $(1,64), .205^{\star \star *}$ & {$[.157, .251]$} & 0.435 & $1.21 \times 10^{9}( \pm 1.03 \%)$ \\
\hline \multirow[t]{4}{*}{ Reward } & 1 & $(1,122),-.168^{\star \star \star}$ & {$[-.191,-.145]$} & -0.336 & $4.88 \times 10^{5}( \pm 1.09 \%)$ \\
\hline & 2 & $(1,237),-.081^{\star * *}$ & {$[-.107,-.055]$} & -0.176 & $206.86( \pm 1.15 \%)$ \\
\hline & 3 & $(1,88),-.253^{\star \star \star}$ & {$[-.317,-.187]$} & -0.482 & $3.06 \times 10^{4}( \pm 1.29 \%)$ \\
\hline & 4 & $(1,64),-.345^{\star * \star}$ & {$[-.399,-.291]$} & -0.738 & $1.06 \times 10^{16}( \pm 1.34 \%)$ \\
\hline \multirow{4}{*}{$\begin{array}{l}\text { Cognitive Load x } \\
\text { Reward }\end{array}$} & 1 & $(1,122),-.050^{\star * *}$ & {$[-.069,-.037]$} & - & $2.32 \times 10^{5}( \pm 3.16 \%)$ \\
\hline & 2 & $(1,237),-.026^{\star \star *}$ & {$[-.036,-.017]$} & - & $2.35 \times 10^{9}( \pm 1.54 \%)$ \\
\hline & 3 & $(1,88),-.092^{\star \star *}$ & {$[-.134,-.049]$} & - & $5.12 \times 10^{26}( \pm 3.75 \%)$ \\
\hline & 4 & $(1,64),-.027$ & {$[-.078, .024]$} & - & $0.18( \pm 3.40 \%)$ \\
\hline
\end{tabular}

Table 2: Influence of cognitive load and reward on STRTs. Cognitive load increased STRTs across all four experiments, whereas reward decreased STRTs across all four experiments. When cognitive load was high, reward had a stronger influence on STRTs than when cognitive load was low. 


\begin{tabular}{|c|c|c|c|c|c|}
\hline Manipulation & Experiment & $(\mathrm{DF}), \beta$ & $95 \% \mathrm{CI}$ & Cohen's $d$ & $\mathrm{BF}_{10}$ \\
\hline \multirow[t]{4}{*}{ Cognitive Load } & 1 & $(1,122),-.368^{\star \star \star}$ & {$[-.401,-.335]$} & -0.779 & $2.58 \times 10^{38}( \pm 1.05 \%)$ \\
\hline & 2 & $(1,237),-.459^{* \star *}$ & {$[-.487,-.432]$} & -0.992 & $1.76 \times 10^{85}( \pm 2.02 \%)$ \\
\hline & 3 & $(1,88),-.344^{* * *}$ & {$[-.387,-.302]$} & -0.731 & $1.97 \times 10^{23}( \pm 0.89 \%)$ \\
\hline & 4 & $(1,64),-.511^{\star * *}$ & {$[-.568,-.455]$} & -1.14 & $5.25 \times 10^{22}( \pm 1.12 \%)$ \\
\hline \multirow[t]{2}{*}{ Reward } & 3 & $(1,91),-.040^{\star}$ & {$[-.074,-.006]$} & -0.073 & $0.36( \pm 0.96 \%)$ \\
\hline & 4 & $(1,64),-.086^{\star *}$ & {$[-.137,-.036]$} & -0.184 & $6.20( \pm 1.70 \%)$ \\
\hline \multirow{2}{*}{$\begin{array}{l}\text { Cognitive Load x } \\
\text { Reward }\end{array}$} & 3 & $(1,88),-.711^{* *}$ & {$[-1.13,-.292]$} & - & $3.01 \times 10^{9}( \pm 2.81 \%)$ \\
\hline & 4 & $(1,64),-.670^{* *}$ & {$[-1.11,-.232]$} & - & $1.86 \times 10^{13}( \pm 3.01 \%)$ \\
\hline
\end{tabular}

\section{General Discussion}

In four experiments, we showed that attention both within and between concurrent media processing tasks is driven by the balance of reward and cognitive load (effort required to execute the task) between each of the available tasks. When the secondary task was more rewarding, it "captured" more attentional resources, reflected in faster RTs to high reward probes (supporting H1). When cognitive load was high, task performance was reduced in both the primary and the secondary task across all four experiments (reflective of reduced attentional resource availability; supporting H2). Furthermore, the gap in RTs between high- and low- reward probes was larger when cognitive load was high (supporting H3). Finally, increasing the rewardingness of one task led to a decrease in attentional resource allocation to other tasks, and this effect was also magnified by cognitive load. Finally, results suggest that when a rewarding secondary task requires enough attentional resources to preclude effective performance of both tasks, the primary task is likely to be abandoned in favor of the secondary task (supporting H4).

\section{Motivated multitasking}

Previous work (e.g, Lang et al., 2006) proposed that variation in media processing performance and/or secondary task reaction times (STRTs) during message processing are an indicator of a change in resources available (the resources allocated to the message minus the resources required by the message; Lang et al., 2006; Lang \& Basil, 1998). The results presented here indicate that this proposition can be extended to allow for the idea that resources can be directly allocated to multiple concurrent tasks, rather than needing to pass through the primary task. As such, changes in performance within any concurrently performed task, reflect either: a) a change in the overall availability of cognitive resources (e.g. due to changes in the resource requirements of a given task), or b) a change in the prioritization of that task relative to other available tasks. This theoretical rationale aligns with recent work from cognate fields demonstrating that attention within and between tasks is guided by neural competition between potential resource allocation targets (Itti \& Koch, 2001; Theeuwes, 2010; Zelinsky \& Bisley, 2015), and with empirical evidence that the ability of a given stimulus to elicit attention is contingent on current cognitive effort requirements (Watson et al., 2019), and on the context in which the stimulus occurs (Otto \& Vassena, 2020).

In providing support for the idea of multiple concurrent attentional loci during message processing, these results also build a bridge between models of attention to individual media processing tasks (like the LC4MP) and models of how people direct their attention across multiple concurrent media 
processing tasks-most notably the "threaded cognition" model (David, 2017; Salvucci \& Taatgen, 2008) and the multiplex model (Feng et al., 2014). In this study, we showed that attentional resource allocation among multiple concurrent media tasks is driven by characteristics of each of the tasks-namely their rewardingness and the cognitive effort that is required to engage in them. These findings stand to assist researchers in incorporating motivational considerations within extant multitasking models, seeking to address not only how people multitask with media, but also why people choose to switch among a given set of media tasks. The potential of future work in this area is especially salient in view of extant efforts to formally model task-switching behaviors in a media context (Fisher \& Hamilton, 2021; Wang et al., 2011), and to understand when visual and auditory media stimuli rely on the same resources, and when they rely on separable resource pools (Fisher et al., 2019).

\section{Overload or offload?}

Media scholars have long been interested in the factors that drive individuals to direct their attention away from a particular mediated message and toward something else. One body of work has shown that large increases in the complexity of a message processing task are associated with a precipitous decline in message processing performance, and often with increased performance in a secondary task (Fox et al., 2007). In the same vein, messages that induce affective conflict (e.g. a highly negative message paired with appetitive cues) can elicit similar cognitive patterns-decreased performance in the message processing task and increased performance in a secondary task (Clayton et al., 2018, 2020; Liu \& Bailey, 2018).

The results presented herein provide a hint as to a common mechanism that may be at play in the attention shifting patterns observed during both cognitive overload and defensive processing. As the crystal collection ("primary") task increased in cognitive load, participants preferentially allocated resources to more rewarding alternative tasks (responding to high-value RT probes). When cognitive load in the crystal collection task was high, and all of the probes were highly rewarding, participants allocated resources away from the primary task and toward the alternative task-especially in Experiment 4 when the alternative task was most intrusive. This hints at the possibility that a common, cognitive offloading process driven by shifts in the relative priority of available tasks may underlie both cognitive overload and defensive processing. Lending credence to this possibility, recent work by Clayton and colleagues (Clayton et al., 2018,2020 ) suggests that individual differences in motivational reactivity predict the alternative task that a person chooses when allocating resources away from a message. Individuals who were low in motivational reactivity tended to avoid the message and allocate resources toward the secondary task, but those high in motivational reactivity tended to engage in counter-arguing, leading to reduced performance in both the primary and secondary tasks.

\section{Limitations and future directions}

The implications of the experimental results presented herein should be considered in view of two primary limitations. First, this study used a very direct and straightforward manipulation of reward (in-game points) that was clearly communicated to the participants. It is clear that the associative learning processes involved in assigning value to everyday stimuli and tasks are much noisier, and that the rewardingness of certain behaviors in the real world are rarely directly advertised. Indeed, reward is notoriously multifarious and contextual (Juechems \& Summerfield, 2019), as are the "costs" by which reward is discounted (Inzlicht et al., 2018; Shenhav et al., 2017). Although converging evidence indicates that these concepts are instantiated in the brain via context-independent neural signals (Hauser et al., 2017; Levy \& Glimcher, 2012; Rushworth et al., 2011), the exact nature of the connection(s) between neural responses and reward-driven behavior in the real world is still very much a science in development.

Second, this study only directly manipulated reward in the "secondary" (RT) task, and only manipulated cognitive load in the "primary" (crystal collection) task, although the theoretical model employed herein eschews the idea of a strict hierarchy between primary and secondary (or tertiary, etc.) tasks. Future research should seek to manipulate reward and load across all concurrent tasks and test the effect of rewardcost/effort ratios among all concurrent tasks. Relatedly, the two tasks employed in these experiments were somewhat different from one another, potentially introducing confounds related to sensorimotor 
processes or intrinsic motivation between the tasks. Future efforts should aim to maximize the functional equivalence between concurrent tasks in view of optimally controlling the costs and rewards of each.

Finally, this study manipulated cognitive load and reward in a binary fashion (high/low load, high/low reward), limiting the informativeness of these results for inferring the parametric relationship between reward and effort requirements as it relates to attentional priority. Extant work suggests that attention networks in the brain respond to increased cognitive demands in a nonlinear fashion (Weber et al., 2018), exhibiting a pattern of initial robustness to perturbation followed by a rapid transition into different states. Manipulating reward and load along a continuum will allow for a deeper understanding of how the human processing system chooses among multiple alternatives within a media environment, as well as how these processes may be influenced by individual differences and attention-related cognitive disorders like attention/deficit hyperactivity disorder (ADHD).

\section{Conclusion}

Using a custom-developed video game stimulus, across four experiments, we showed that attention within and between multiple concurrent media tasks can be explained in terms of a cognitive "priority map" that is shaped by the rewardingness and cognitive effort requirements of available tasks. Using both reaction time and performance measures, we found that increased cognitive load in one task leads to reduced performance across concurrent tasks, that increased reward leads to increased attention to the rewarded task, and that the attention-capturing influence of rewarding tasks is magnified under high cognitive load. These results provide support for proposed updates to LC4MP which modify its description of the resource allocation process in a way that allows it to account for the interacting influence of multiple concurrent tasks. This work also serves as a foundation for the development of integrative frameworks for understanding how people direct their attention among multiple concurrent media tasks and suggest a common mechanism undergirding frequently observed phenomena in communication and media research such as cognitive overload and defensive processing.

\section{References}

Anderson, B. (2011). There is no such thing as attention. Frontiers in Psychology, 2(146), 1-8. https://doi.org/10.3389/ fpsyg.2011.00246

Anderson, B. A. (2016). The attention habit: How reward learning shapes attentional selection. Annals of the New York Academy of Sciences, 1369(1), 24-39. https://doi. org/10.1111/nyas.12957

Anderson, B. A., Laurent, P. A., \& Yantis, S. (2011). Valuedriven attentional capture. Proceedings of the National Academy of Sciences, 108(25), 10367-10371. https://doi. org/10.1073/pnas.1104047108

Awh, E., Belopolsky, A. V., \& Theeuwes, J. (2012). Top-down versus bottom-up attentional control: A failed theoretical dichotomy. Trends in Cognitive Sciences, 16(8), 437-443. https://doi.org/10.1016/j.tics.2012.06.010

Awh, E., \& Pashler, H. (2000). Evidence for split attentional foci. Journal of Experimental Psychology: Human Perception and Performance, 26(2), 834-846. https://doi. org/10.1037/0096-1523.26.2.834

Baddeley, A. D., \& Hitch, G. J. (1974). Working memory. In G. H. Bower (Ed.), Psychology of Learning Motivation (pp. 48-79).

Bates, D., Mächler, M., Bolker, B., \& Walker, S. (2015). Fitting linear mixed-effects models using Ime4. Journal of Statistical Software, 67(1), 1-48. https://doi.org/10.18637/jss.v067.i01
Behrens, T. E. J., Muller, T. H., Whittington, J. C. R., Mark, S., Baram, A. B., Stachenfeld, K. L., \& Kurth-Nelson, Z. (2018). What is a cognitive map? Organizing knowledge for flexible behavior. Neuron, 100(2), 490-509. https://doi.org/10.1016/j. neuron.2018.10.002

Bisley, J. W., \& Mirpour, K. (2019). The neural instantiation of a priority map. Current Opinion in Psychology, 29, 108-112. https://doi.org/10.1016/j.copsyc.2019.01.002

Broadbent, D. E. (1958). Perception and communication. Pergamon Press.

Cartier, F. A., \& Harwood, K. A. (1958). Some questions about attention. Journal of Communication, 8, 106-110.

Chaffee, S. H., \& Berger, C. R. (1987). What communication scientists do. In C. R. Berger \& S. H. Chaffee (Eds.), Handbook of communication science (pp. 99-122). SAGE.

Chelazzi, L., Eštočinová, J., Calletti, R., Gerfo, E. L., Sani, I., Libera, C. D., \& Santandrea, E. (2014). Altering spatial priority maps via reward-based learning. Journal of Neuroscience, 34(25), 8594-8604. https://doi.org/10.1523/ JNEUROSCI.0277-14.2014

Chelazzi, L., \& Santandrea, E. (2018). The time constant of attentional control: Short, medium and long (infinite?). Journal of Cognition, 1(1), 27. https://doi.org/10.5334/joc.24 
Clayton, R. B., Lang, A., Leshner, G., \& Quick, B. L. (2018). Who fights, who flees? An integration of the LC4MP and psychological reactance theory. Media Psychology, 22, 545-571. https://doi.org/10.1080/15213269.2018.1476157

Clayton, R. B., Leshner, G., Sanders-Jackson, A., \& Hendrickse, J. (2020). When counterarguing becomes the primary task: Examination of dogmatic anti-vaping messages on psychological reactance, available cognitive resources, and memory. Journal of Communication, 70(4), 522-547. https://doi.org/10.1093/joc/jqaa010

Corbetta, M., \& Shulman, G. L. (2002). Control of goaldirected and stimulus-driven attention in the brain. Nature Reviews Neuroscience, 3(3), 201-215. https://doi. org/10.1038/nrn755

David, P. (2017). Threaded cognition approach to multitasking and activity switching in a permanently online and permanently connected ecosystem. In P. Vorderer, D. Hefner, L. Reinecke, \& C. Klimmt (Eds.), Permanently Online, Permanently Connected: Living and Communicating in a POPC World (pp. 83-95). Routledge.

Deloitte. (2017). Digital democracy survey: A multigenerational view of consumer technology, media, and telecom trends.

Desimone, R., \& Duncan, J. (1995). Neural mechanisms of selective visual attention. Annual Review of Neuroscience, 18(1), 193-222.

Eriksen, C. W., \& St. James, J. D. (1986). Visual attention within and around the field of focal attention: A zoom lens model. Perception \& Psychophysics, 40(4), 225-240. https:// doi.org/10.3758/BF03211502

Failing, M., \& Theeuwes, J. (2018). Selection history: How reward modulates selectivity of visual attention. Psychonomic Bulletin \& Review, 25(2), 514-538. https://doi. org/10.3758/s13423-017-1380-y

Fecteau, J. H., \& Munoz, D. P. (2006). Salience, relevance, and firing: A priority map for target selection. Trends in Cognitive Sciences, 10(8), 382-390. https://doi.org/10.1016/j. tics.2006.06.011

Feng, S. F., Schwemmer, M., Gershman, S. J., \& Cohen, J. D. (2014). Multitasking versus multiplexing: Toward a normative account of limitations in the simultaneous execution of control-demanding behaviors. Cognitive, Affective, \& Behavioral Neuroscience, 14(1), 129-146. https://doi. org/10.3758/s13415-013-0236-9

Fine, M. S., \& Minnery, B. S. (2009). Visual salience affects performance in a working memory task. Journal of Neuroscience, 29(25), 8016-8021. https://doi.org/10.1523/ JNEUROSCI.5503-08.2009

Fisher, J. T., Huskey, R., Keene, J. R., \& Weber, R. (2018). The limited capacity model of motivated mediated message processing: Looking to the future. Annals of the International Communication Association, 42(4), 291-315. https://doi.org/ $10.1080 / 23808985.2018 .1534551$
Fisher, J. T., Keene, J. R., Huskey, R., \& Weber, R. (2018). The limited capacity model of motivated mediated message processing: Taking stock of the past. Annals of the International Communication Association, 42(4), 270-290. https://doi.org/10.1080/23808985.2018.1534552

Fisher, J. T., Hopp, F. R., \& Weber, R. (2019). Modality-specific effects of perceptual load in multimedia processing. Media \& Communication, 7(4), 149-165. https://doi.org/10.33767/osf. io/cz2pj

Fisher, J. T., \& Hamilton, K. (2021). Integrating media selection and media effects using decision theory. Journal of Media Psychology. 33, 215-225. https://doi.org/10.33767/osf. io/pseza

Floresco, S. B., Onge, J. R. St., Ghods-Sharifi, S., \& Winstanley, C. A. (2008). Cortico-limbic-striatal circuits subserving different forms of cost-benefit decision making. Cognitive, Affective, \& Behavioral Neuroscience, 8(4), 375-389. https:// doi.org/10.3758/CABN.8.4.375

Fox, J. R., Park, B., \& Lang, A. (2007). When available resources become negative resources: The effects of cognitive overload on memory sensitivity and criterion bias. Communication Research, 34(3), 277-296. https://doi. org/10.1177/0093650207300429

Gottlieb, J., \& Balan, P. (2010). Attention as a decision in information space. Trends in Cognitive Sciences, 14(6), 240-248. https://doi.org/10.1016/j.tics.2010.03.001

Grabenhorst, F., \& Rolls, E. T. (2011). Value, pleasure and choice in the ventral prefrontal cortex. Trends in Cognitive Sciences, 15(2), 56-67. https://doi.org/10.1016/j. tics.2010.12.004

Green, P., \& MacLeod, C. J. (2016). SIMR: An R package for power analysis of generalized linear mixed models by simulation. Methods in Ecology and Evolution, 7(4), 493-498. https://doi.org/10.1111/2041-210X.12504

Gu, R., Huang, W., Camilleri, J., Xu, P., Wei, P., Eickhoff, S. B., \& Feng, C. (2019). Love is analogous to money in human brain: Coordinate-based and functional connectivity meta-analyses of social and monetary reward anticipation. Neuroscience \& Biobehavioral Reviews, 100, 108-128. https://doi. org/10.1016/j.neubiorev.2019.02.017

Hasher, L., \& Zacks, R. T. (1979). Automatic and effortful processes in memory. Journal of Experimental Psychology: General, 108(3), 356-388.

Hauser, T. U., Eldar, E., \& Dolan, R. J. (2017). Separate mesocortical and mesolimbic pathways encode effort and reward learning signals. Proceedings of the National Academy of Sciences, 114(35), E7395-E7404. https://doi. org/10.1073/pnas.1705643114

Inzlicht, M., Shenhav, A., \& Olivola, C. Y. (2018). The effort paradox: Effort is both costly and valued. Trends in Cognitive Sciences, 22(4), 337-349. https://doi.org/10.1016/j. tics.2018.01.007 
Itti, L., \& Koch, C. (2001). Computational modelling of visual attention. Nature Reviews Neuroscience, 2(3), 194-203. https://doi.org/10.1038/35058500

Juechems, K., \& Summerfield, C. (2019). Where does value come from? Trends in Cognitive Sciences, 23(10), 836-850. https://doi.org/10.1016/j.tics.2019.07.012

Kobayashi, K., \& Hsu, M. (2019). Common neural code for reward and information value. Proceedings of the National Academy of Sciences, 116, 13061-13066. https://doi. org/10.1073/pnas.1820145116

Krauzlis, R. J., Bollimunta, A., Arcizet, F., \& Wang, L. (2014). Attention as an effect not a cause. Trends in Cognitive Sciences, 18(9), 457-464. https://doi.org/10.1016/j. tics.2014.05.008

Lang, A. (2000). The limited capacity model of mediated message processing. Journal of Communication, 50(1), 46-70. https://doi.org/10.1111/j.1460-2466.2000.tb02833.x

Lang, A. (2009). The limited capacity model of motivated mediated message processing. In R. Nabi \& M. B. Oliver (Eds.), The SAGE Handbook of Media Processes and Effects (pp. 193-204). SAGE.

Lang, A., \& Basil, M. D. (1998). Attention, resource allocation, and communication research: What do secondary task reaction times measure, anyway? Annals of the International Communication Association, 21(1), 443-458. https://doi.org/ 10.1080/23808985.1998.11678957

Lang, A., Bradley, S. D., Park, B., Shin, M., \& Chung, Y. (2006). Parsing the resource pie: Using STRTs to measure attention to mediated messages. Media Psychology, 8(4), 369-394.

Lang, A., \& Ewoldsen, D. (2009). Beyond effects: Conceptualizing communication as dynamics, complex, nonlinear, and fundamental. In S. Allen (Ed.), Rethinking Communication: Keywords in Communication Research (pp. 109-120). Hampton Press.

Levy, D. J., \& Glimcher, P. W. (2012). The root of all value: A neural common currency for choice. Current Opinion in Neurobiology, 22(6), 1027-1038. https://doi.org/10.1016/j. conb.2012.06.001

Liu, J., \& Bailey, R. L. (2018). Effects of substance cues in negative public service announcements on cognitive processing. Health Communication, 34, 964-974. https://doi. org/10.1080/10410236.2018.1446251

McMains, S. A., \& Somers, D. C. (2004). Multiple spotlights of attentional selection in human visual cortex. Neuron, 42(4), 677-686. https://doi.org/10.1016/S0896-6273(04)00263-6

Morey, R. D., Romeijn, J.-W., \& Rouder, J. N. (2016). The philosophy of Bayes factors and the quantification of statistical evidence. Journal of Mathematical Psychology, 72, 6-18. https://doi.org/10.1016/j.jmp.2015.11.001

Morey, R. D., Rouder, J. N., Jamil, T., Urbanek, S., Forner, K., \& Ly, A. (2018). BayesFactor: Computation of Bayes factors for common designs (0.9.12-4.2) [Computer software]. https:// CRAN.R-project.org/package=BayesFactor
Otto, A. R., \& Vassena, E. (2020). It's all relative: Rewardinduced cognitive control modulation depends on context. Journal of Experimental Psychology: General. https://doi. org/10.1037/xge0000842

Posner, M. I., Snyder, C. R., \& Davidson, B. J. (1980). Attention and the detection of signals. Journal of Experimental Psychology: General, 109(2), 160-174. https://doi. org/10.1037/0096-3445.109.2.160

Ptak, R. (2012). The frontoparietal attention network of the human brain: Action, saliency, and a priority map of the environment. The Neuroscientist, 18(5), 502-515. https://doi. org/10.1177/1073858411409051

R Core Team. (2013). R: a language and environment for statistical computing. R Foundation for Statistical Computing. http://www.R-project.org/

Rains, S. A., Levine, T. R., \& Weber, R. (2018). Sixty years of quantitative communication research summarized: Lessons from 149 meta-analyses. Annals of the International Communication Association, 42(2), 105-124. https://doi.org/ $10.1080 / 23808985.2018 .1446350$

Rangel, A., \& Hare, T. (2010). Neural computations associated with goal-directed choice. Current Opinion in Neurobiology, 20(2), 262-270. https://doi.org/10.1016/j.conb.2010.03.001

Rouder, J. N., Morey, R. D., Verhagen, J., Swagman, A. R., \& Wagenmakers, E.-J. (2017). Bayesian analysis of factorial designs. Psychological Methods, 22(2), 304-321. http:// dx.doi.org/10.1037/met0000057

Rushworth, M. F. S., Noonan, M. P., Boorman, E. D., Walton, M. E., \& Behrens, T. E. (2011). Frontal cortex and rewardguided learning and decision-making. Neuron, 70(6), 1054-1069. https://doi.org/10.1016/j.neuron.2011.05.014

Salvucci, D. D., \& Taatgen, N. A. (2008). Threaded cognition: An integrated theory of concurrent multitasking. Psychological Review, 115(1), 101-130. https://doi. org/10.1037/0033-295X.115.1.101

Segijn, C. M., Xiong, S., \& Duff, B. R. L. (2018). Manipulating and measuring media multitasking: Implications of previous research and guidelines for future research. Communication Methods and Measures, 13(2), 83-101. https://doi.org/10.108 0/19312458.2018.1555797

Shenhav, A., Botvinick, M. M., \& Cohen, J. D. (2013). The expected value of control: An integrative theory of anterior cingulate cortex function. Neuron, 79(2), 217-240. https:// doi.org/10.1016/j.neuron.2013.07.007

Shenhav, A., Musslick, S., Lieder, F., Kool, W., Griffiths, T. L., Cohen, J. D., \& Botvinick, M. M. (2017). Toward a rational and mechanistic account of mental effort. Annual Review of Neuroscience, 40(1), 99-124. https://doi.org/10.1146/ annurev-neuro-072116-031526 
Shiffrin, R. M., \& Schneider, W. (1977). Controlled and automatic human information processing: II. Perceptual learning, automatic attending and a general theory. Psychological Review, 84(2), 127-190. https://doi. org/10.1037/0033-295X.84.2.127

Simons, D. J., \& Chabris, C. F. (1999). Gorillas in our midst: Sustained inattentional blindness for dynamic events. Perception, 28(9), 1059-1074. https://doi.org/10.1068/ p281059

Theeuwes, J. (2010). Top-down and bottom-up control of visual selection. Acta Psychologica, 135(2), 77-99. https:// doi.org/10.1016/j.actpsy.2010.02.006

Theeuwes, J. (2019). Goal-driven, stimulus-driven, and history-driven selection. Current Opinion in Psychology, 29, 97-101. https://doi.org/10.1016/j.copsyc.2018.12.024

Veltman, D. J., Rombouts, S. A. R. B., \& Dolan, R. J. (2003). Maintenance versus manipulation in verbal working memory revisited: An fMRI study. Neurolmage, 18(2), 247-256. https:// doi.org/10.1016/S1053-8119(02)00049-6

Wang, Z. J., Irwin, M., Cooper, C., \& Srivastava, J. (2015). Multidimensions of media multitasking and adaptive media selection. Human Communication Research, 41(1), 102-127.

Wang, Z. J., Lang, A., \& Busemeyer, J. R. (2011). Motivational processing and choice behavior during television viewing: An integrative dynamic approach. Journal of Communication, 61(1), 71-93. https://doi.org/10.1111/ j.1460-2466.2010.01527.x

Watson, P., Pearson, D., Chow, M., Theeuwes, J., Wiers, R. W., Most, S. B., \& Le Pelley, M. E. (2019). Capture and control: Working memory modulates attentional capture by rewardrelated stimuli. Psychological Science, 30(8), 1174-1185. https://doi.org/10.1177/0956797619855964

Weber, R., Alicea, B., Huskey, R., \& Mathiak, K. (2018). Network dynamics of attention during a naturalistic behavioral paradigm. Frontiers in Human Neuroscience, 12(182), 1-14. https://doi.org/10.3389/fnhum.2018.00182

Weber, R., Mathiak, K., \& Sherry, J. L. (2009). The neurophysiological perspective in mass communication research. In M. Beatty, J. McCroskey, \& K. Floyd (Eds.), Biological dimensions of communication: Perspectives, methods, and research (pp. 43-73). Hampton Press.

Westbrook, A., \& Braver, T. S. (2015). Cognitive effort: A neuroeconomic approach. Cognitive, Affective, \& Behavioral Neuroscience, 15(2), 395-415. https://doi.org/10.3758/ s13415-015-0334-y

Wilson, R. C., Takahashi, Y. K., Schoenbaum, G., \& Niv, Y. (2014). Orbitofrontal cortex as a cognitive map of task space. Neuron, 81(2), 267-279. https://doi.org/10.1016/j. neuron.2013.11.005
Zelinsky, G. J., \& Bisley, J. W. (2015). The what, where, and why of priority maps and their interactions with visual working memory. Annals of the New York Academy of Sciences, 1339(1), 154-164. https://doi.org/10.1111/ nyas. 12606 


\section{Experiment 1}

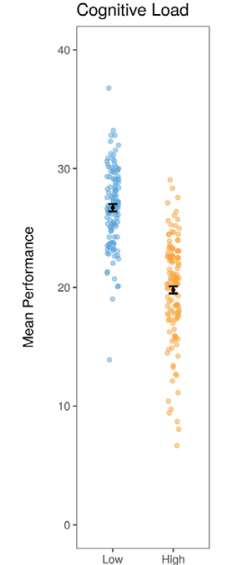

a.

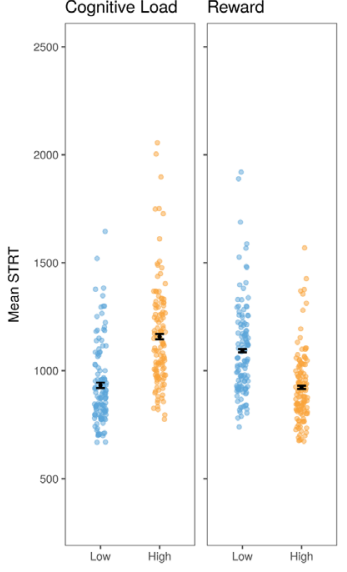

b.

c.

\section{Experiment 2}
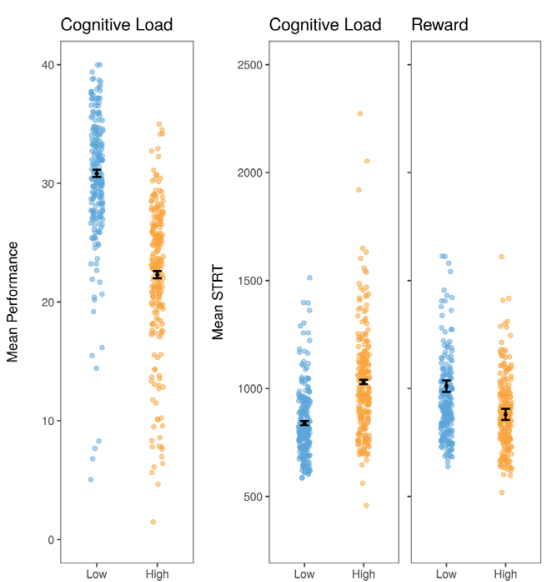

a.

b.

c.

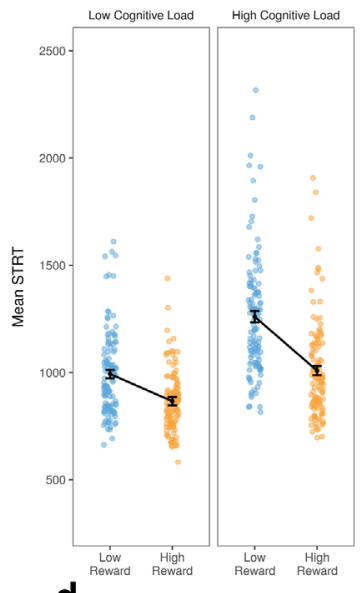

d.

a.
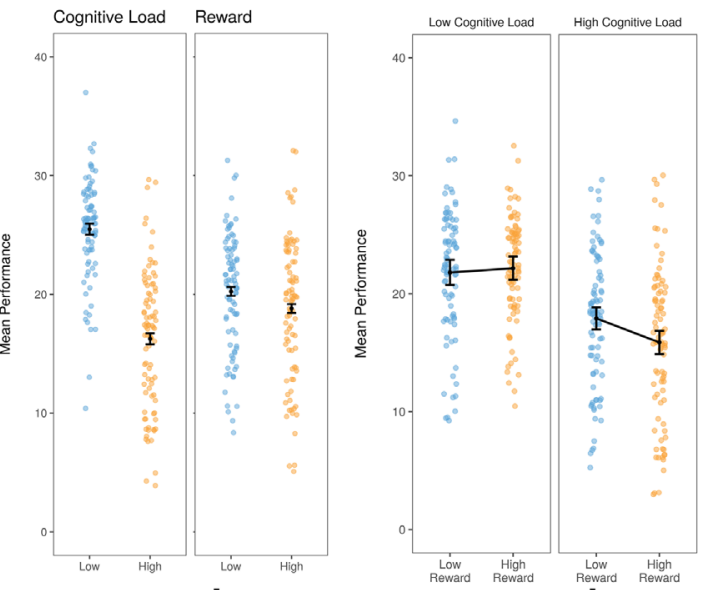

c.

d.

\section{Experiment 4}
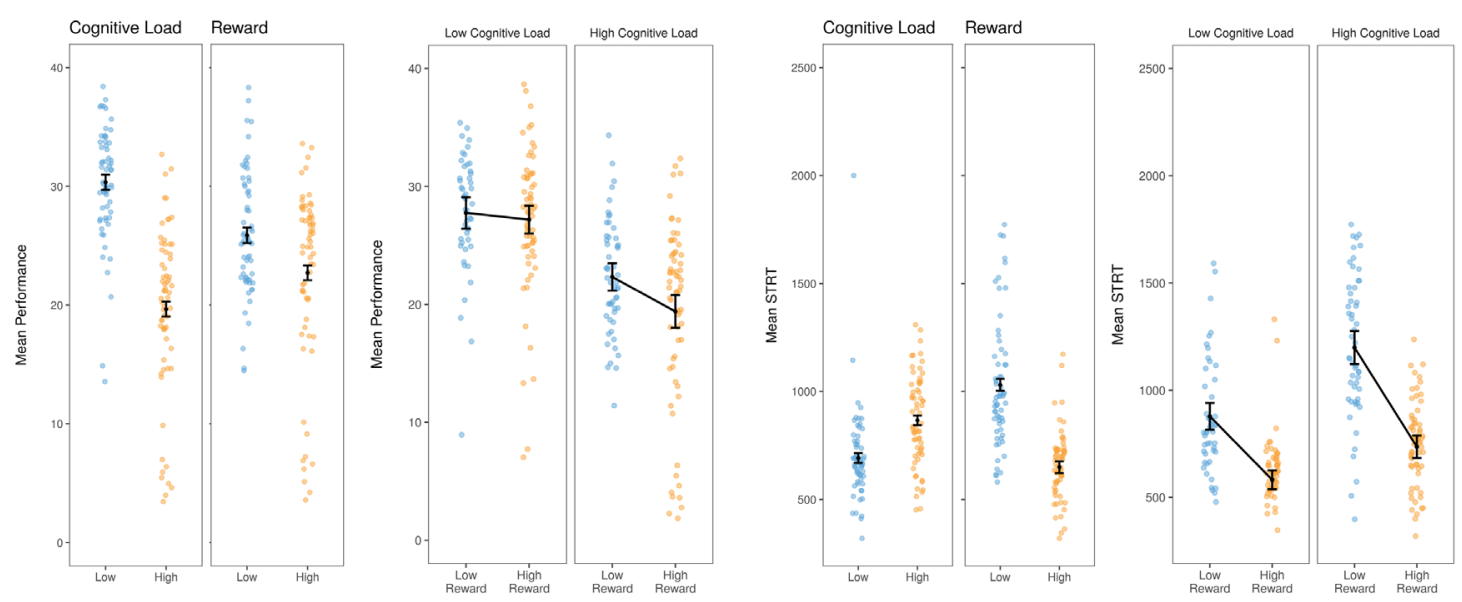

a.

b.

c.

d.
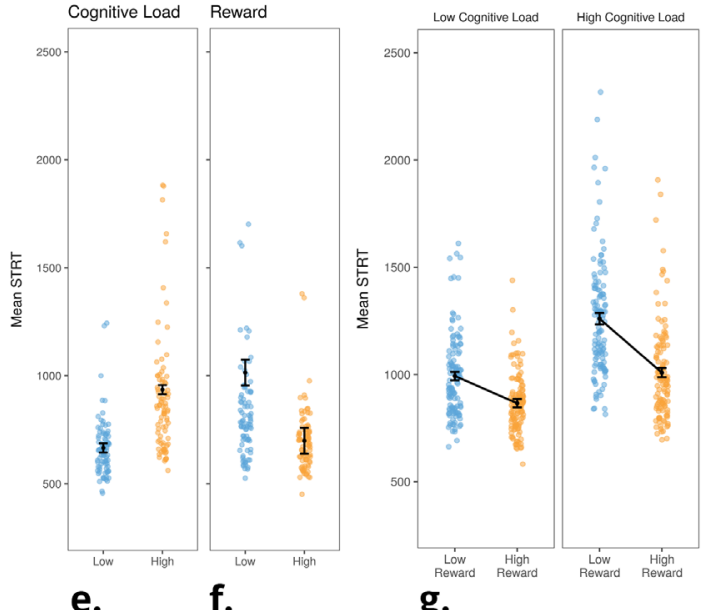

g.

Figure 2: Summary of experimental findings. Across all four experiments, it was shown that: a) increased cognitive effort reduces performance in the primary task (14a), b) increased cognitive effort increases RTs (1b, 2b, 3d, 4d); c) reward reduces RTs (1c, 2c, 3e, 4e); d) effort requirements and reward interact such that the influence of high-reward RTs is magnified when effort requirements are high (1d, 2d, 3f, 4f). Whenever the RT task itself was either high or low reward (Experiments 3 and 4 ), the rewardingness of the RT task influenced performance on the primary task such that performance in the primary task decreased when the RT task was high reward. 